\title{
INTERVIEW
}

For reprint orders, please contact: reprints@futuremedicine.com

\section{Survivor and patient advocate: an interview with T.J. Sharpe}

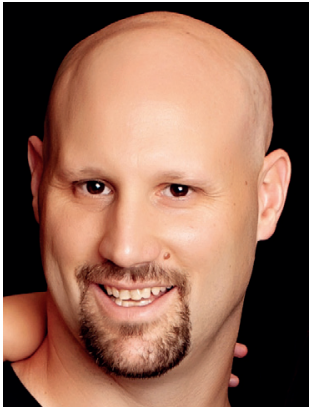

T.J. Sharpe* speaks to Sebastian Dennis-Beron, Commissioning Editor:T.J.Sharpe is astage IV melanoma patient who shares his journey through cancer in the Patient \#1 Blog [1]. He was diagnosed in August 2012 with melanoma tumors in multiple organs; since then, he has undergone six surgeries and four immunotherapy treatments over two different clinical trials. The initial failures and subsequent significantly positive response have been chronicled in his blog posts since January 2013. His story is about life with a serious illness, and the mental and emotional hurdles a patient must clear. He writes how a cancer patient's challenges mirror those many face, and portrays life through the optimistic prism of a patient who is on the long, winding road toward overcoming melanoma's long odds and deadly consequences. He also shares the latest melanoma and oncology research breakthroughs and advocacy events, and initiatives to help others avoid, detect or educate themselves on cancer. As part of his advocacy, he attends cancer and melanoma conferences, reporting on the latest medical breakthroughs through his blog to other cancer patients. He also partners with advocacy organizations, clinical trial groups and the pharmaceutical industry to bring awareness to cancer research and developments. His efforts have been recognized by several organizations, including the Melanoma Research Foundation, Melanoma International Foundation, Patient Power, Forbes, Merck, GlaxoSmithKline, the Drug Information Association and the Milken Institute's FasterCures. In December 2014, he was able to share his story on Capitol Hill in a special session promoting immunotherapy research to lawmakers. A South Jersey native, T.J. lives in Fort Lauderdale with his wife Jennifer and two young children, Josie and Tommy. He is active in health and wellness initiatives, including melanoma/cancer awareness runs and bike rides, and an avid yoga participant. $\mathrm{He}$ also serves on the Board of Directors of A Prom to Remember, a 501(c)3 charity providing pediatric oncology teens with the ultimate prom experience.

Accepted for publication: 19 April 2017; Published online: 31 July 2017

Q Could you tell our readers a little about your life prior to your diagnosis?

I was an IT Project Manager with a 2-yearold daughter and a newborn son. We lived a very active life in Fort Lauderdale, FL, USA. Despite an earlier, early stage melanoma diagnosis when I was 25, it never really affected our lives. We spent less time 'in the sun' but still were very active outdoors; just protected a little (or a lot) better. I also volunteered on the Board for a charity for teens affected by cancer called A Prom To Remember. My prior brush with cancer had given me a better perspective and admiration for those kids; little did I know that I would soon be in treatment as well.

How did you react to your melanoma diagnosis in 2012; how has the diagnosis changed your life?

I was determined to find the best chance of getting a durable response. It was tough to

\section{KEYWORDS}

- advocacy • clinical trials

- immunotherapy • melanoma

- patient care 
hear, but I reacted as best I think I could: understanding the diagnosis and prognosis, finding the best options for care and ultimately making the right decisions to give me that chance at seeing my children grow up.

It has changed everything in one way or another. Physically, it has changed the way me and my family look at being healthy and mindful of being consistent and balanced in our lives. Mentally and emotionally, it has been both very good and very challenging. We have tried over more than 4 years of this battle to address the reality of cancer head on; at the same time, I wanted to ensure our family was able to do as much 'normal' as we can, and to give my children the same upbringing they would get with two healthy parents.

\section{Q Can you give us an outline of your treatment} course?

Here is the summary: two clinical trials with four immunotherapies and six surgeries.

After my initial surgery to remove an $8 \mathrm{~cm}$ tumor, we looked for the systemic treatment that would give me the aforementioned best chance at a long-term response. After several second opinions, we were finally offered a clinical trial (two, actually) and chose to do an immunotherapy combination that used a recently approved antiCTLA-4 checkpoint inhibitor (ipilimumab, aka Yervoy $^{\circledR}$, Bristol-Myers Squibb, NY, USA) and an experimental type of adoptive cell transfer called tumor-infiltrating lymphocytes (TIL), which takes $T$ cells out of one of your tumors, finds the ones that show the most promise against those specific tumors and then exponentially grows those T cells into billions and re-infuses them. After the infusion, high doses of IL-2 are given to support T-cell growth and proliferation. This TIL/IL-2 regiment was placed in between doses two and three of the Yervoy treatment (which has four total doses).

When the tumors continued to grow at the conclusion of that study, I was able to enroll in an antiPD-1 trial, and the results were almost immediate. Four years later, I have gotten a partial response with stable disease because there are still two spots on CT scans; however, with no cancer detectable in PET scans, I believe that I am cancer free.

Along the way, there were six different surgeries; the initial tumor removal, a lower-lung resection (to get the tumor for the TIL/T cells), a colostomy (bad luck, when there was a diverticulitis during trial \#1), tonsil removal (tumor), adrenal gland removal (a tumor cropped up during trial \#2) and finally, colostomy reversal.
Q What did you feel were some of the best aspects of your care as a patient? Conversely, are there aspects where you feel your needs were not sufficiently met \& could be improved on? Being on a trial has, for the most part, given me the best possible standard of care. There were many times where I felt like I was the most important patient to my medical team: quick responses at any and all hours, personal attention and genuine concern for my health and well-being.

There were some parts that were less positive. The second trial was simply not as good an experience as I had envisioned; as someone who was very much in charge of my own health, this led to differences with the group. I would have also hoped for a more knowledgeable team, though they have since moved on from the hospital, and my new team is great. Still, it highlighted the experience that happens for many patients and some of those will not know to push back or insist on the best possible care.

Q What was your experience like over the two different immunotherapy clinical trials you took part in?

My immune system went for a roller coaster ride. There were some side effects that are typical for immunotherapies; however, none were severe or unbearable, and my overall quality of life during my treatments was almost always better than the standard of care chemotherapy options. It also let me have a very small amount of control over my treatment as well; one doctor told me "You are using your immune system to beat cancer; you should give it every chance to do its job." So I modified my diet, sleep and lifestyle to focus on being healthy without going overboard. We can never know if there was any effect on my recovery, but just being able to control something was a bit empowering at a time when there were so many things out of our hands.

\section{Q How would you say patient care has developed since entering this new era of immunotherapies for melanoma?}

It is getting exponentially better. The sheer amount of attention that the immunotherapies (and targeted therapies) have received has made more oncologists aware that this is the up and coming standard of care; when I was first diagnosed, that distribution of information was much more limited. I have also seen a better patient experience during interactions with advocacy and industry, as each stakeholder continues to 
adapt to the rise in immunotherapies and patient involvement in their own treatments.

\section{Q In 2014 you were invited to speak on} Capitol Hill to a group of lawmakers on the importance of immune-oncology; how vital is it for the patient voice to be heard at all levels of healthcare?

Having the patient speak to each level of healthcare stakeholders closes the loop for the most impacted persons involved in healthcare: the consumers of medical products and services. For some reason, this is one of the few industries that have erected barriers to open communication and collaboration between providers and consumers. While some of these safety measures are well intentioned, it has created an environment where patients can often feel shut down or shut out of some of the most important decisions they will ever make, their health. Representing patients of all disease types and demographics is a challenge, but extremely rewarding when those stakeholders understand the challenges that are common across patient groups.

Q Is enough being done to engage \& educate patients on the benefits of immunotherapy?

Mostly yes; there is a significant push by industry and advocacy organizations to provide the most up-to-date and comprehensive information. There are reams of information available online, and there has been a good deal of effort put into getting this information out.

There are a few areas of opportunity. First, there is almost an overwhelming amount of information, so navigating to the right information sometimes proves to be tricky. Second, the medium for the most up-to-date information is mostly online, which does miss some of the patients who are less digitally adept. Last, the best place for advancing immunotherapy information is to educate the medical professionals who are involved in the initial diagnosis.

\section{Q Can you tell us about some of your} work as an advocate, such as your work with organizations \& your own blog? How important is this advocacy, not only for the medical field, but also for current patients overcoming the hurdles they face every day? When I was initially diagnosed, I made a vow that I would help others and pay forward the enormous amount of support I got from friends, family and others who had paved the way for my recovery.
The blog started just telling my story, but has blossomed into a great opportunity to be a voice for patients, both sharing other patient stories, and educating patients on melanoma, cancer research, immunotherapies and clinical research. I know there are - and always will be - another patient out there, sitting in a hospital bed or having just left the oncologist's office with their life forever changed. I have this opportunity - this responsibility - to speak to and for those people, to other patients, to those in industry and advocacy and regulatory arenas. Writing and speaking about living and about my experience is, hopefully, making that difference in the lives of patients that will come after me.

\section{Q How would you like to see melanoma} patient care evolve over the coming years? Melanoma can and should stay on the cutting edge of immunotherapies and targeted therapies. With the amount of work already done in this area, it would be the natural next step to continue to push treatments so that better, more effective, and more personalized medicines are found, so that we patients have continued exponential increase in efficacy and survival.

\section{Q Finally, what is next for you?}

I write three blogs on my experiences as a melanoma patient and about skin cancer [1-3]. I continue to contribute to panels in cancer and clinical trial research, and have begun telling my story in keynotes to pharma and industry leaders. Starting in 2017, I have begun consulting with those same groups to bring more patient focus during drug and trial development and execution, not just in trial initiation but across the entire lifespan of a treatment. And I continue to live life with my family (and write about it) in a way that gives hope to those patients who are about to embark on their own journeys.

\section{Closing statement}

I am the luckiest cancer patient in the world. I was able to get on two different groundbreaking trials with the help and support of numerous family, friends and those who have previously walked the path of stage IV melanoma. I have a response I consider complete. I have a healthy family. I have a voice to the community: the patient community, the industry community and my hometown community. 
I get to tell my story to those whose careers are dedicated to treating and curing melanoma, cancer and other terminal or chronic disease; how many patients would want that opportunity? How many patients who have not had a response wish they could have told their story? It is an awesome, rewarding, humbling chance to make a difference for many. Perhaps it will positively affect someone you love, or someone I love. Or someone we do not know at all. Either way, I get to take the hurdles and opportunities I have, and make a difference in the life of someone else. That makes all the time and trial tribulations merely a step to a much greater purpose.

\section{References}

1 Health - Patient 1. www.philly.com/patient1

2 Novartis Oncology. www.novartisoncology.com

3 SkinCancer.net. https://skincancer.net

\section{Disclaimer}

The opinions expressed in this interview are those of the interviewee and do not necessarily reflect the views of Future Medicine Ltd or any other institutions mentioned in the interview.

Financial \& competing interests disclosure

The interviewee has no relevant affliations or financial involvement with any organization or entity with a financial interest in or financial conflict with the subject matter or materials discussed in the manuscript. This includes employment, consultancies, honoraria, stock ownership or options, expert testimony, grants or patents received or pending, or royalties.

No writing assistance was utilized in the production of this manuscript. 